\title{
MULTILINEAR FRACTIONAL INTEGRAL OPERATORS: A COUNTER-EXAMPLE
}

\author{
Pablo Rocha \\ Universidad Nacional del Sur, Departamento de Matemática \\ Av. Alem 1253 - Bahía Blanca 8000, Buenos Aires, Argentina; pablo.rocha@uns.edu.ar
}

Abstract. By means of a counter-example we show that the multilinear fractional operator $\mathcal{I}_{\gamma}$
$(1<\gamma<2)$ is not bounded from $H^{1}(\mathbf{R}) \times H^{p}(\mathbf{R})$ into $H^{q}(\mathbf{R})$, for $0<p \leq \gamma^{-1}$ and $\frac{1}{q}=1+\frac{1}{p}-\gamma$.

\section{Introduction}

Given positive integers $m, n$ and a real number $0<\gamma<m n$, it is define the multilinear fractional operator $\mathcal{I}_{\gamma}$ by

$$
\mathcal{I}_{\gamma}\left(f_{1}, \ldots, f_{m}\right)(x)=\int_{\left(\mathbf{R}^{n}\right)^{m}} \frac{f_{1}\left(y_{1}\right) \cdots f_{m}\left(y_{m}\right)}{\left(\left|x-y_{1}\right|+\cdots+\left|x-y_{m}\right|\right)^{n m-\gamma}} d y_{1} \cdots d y_{m}, \quad x \in \mathbf{R}^{n} .
$$

Lin and $\mathrm{Lu}$ in [3] proved Hardy space estimates for the multilinear fractional operator $\mathcal{I}_{\gamma}$. More precisely, they proved that if $0<\gamma<n, 0<p_{1}, \ldots, p_{m}, q \leq 1$, and $q$ such that $\frac{1}{q}=\frac{1}{p_{1}}+\cdots+\frac{1}{p_{m}}-\frac{\gamma}{n}>0$, then

$$
\left\|\mathcal{I}_{\gamma}\left(f_{1}, \ldots, f_{m}\right)\right\|_{L^{q}} \leq C\left\|f_{1}\right\|_{H^{p_{1}}} \cdots\left\|f_{m}\right\|_{H^{p_{m}}} .
$$

Recently, Cruz-Uribe, Moen and Nguyen in [1] generalized the result of Lin and Lu to weighted Hardy spaces on the full range $0<\gamma<n m$.

The purpose of this note is to give a counter-example to show that the multilinear fractional operator $\mathcal{I}_{\gamma}$ is not bounded from a product of Hardy spaces into a Hardy space. For them, we consider $n=1, m=2, \gamma=\alpha+1$ with $0<\alpha<1$, so $1<\gamma<2$, $2-\gamma=1-\alpha$ and the multilinear fractional operator $\mathcal{I}_{\alpha+1}$ in this case is given by

$$
\mathcal{I}_{\alpha+1}\left(f_{1}, f_{2}\right)(x)=\iint_{\mathbf{R}^{2}} \frac{f_{1}(s) f_{2}(t)}{(|x-s|+|x-t|)^{1-\alpha}} d s d t, \quad x \in \mathbf{R} .
$$

We will prove that the operator $\mathcal{I}_{\alpha+1}$ is not bounded from $H^{1}(\mathbf{R}) \times H^{p}(\mathbf{R})$ into $H^{q}(\mathbf{R})$, for $0<p \leq(\alpha+1)^{-1}$ and $\frac{1}{q}=\frac{1}{p}-\alpha$.

We briefly recall the definition of Hardy space on $\mathbf{R}^{n}$. The Hardy space $H^{p}\left(\mathbf{R}^{n}\right)$ (for $0<p<\infty$ ) consists of tempered distributions $f \in \mathcal{S}^{\prime}\left(\mathbf{R}^{n}\right)$ such that for some Schwartz function $\varphi$ with $\int \varphi=1$, the maximal operator

$$
\left(\mathcal{M}_{\varphi} f\right)(x)=\sup _{t>0}\left|\left(\varphi_{t} * f\right)(x)\right|
$$

is in $L^{p}\left(\mathbf{R}^{n}\right)$, where $\varphi_{t}(x):=\frac{1}{t^{n}} \varphi\left(\frac{x}{t}\right)$. In this case we define $\|f\|_{H^{p}}:=\left\|\mathcal{M}_{\varphi} f\right\|_{p}$ as the $H^{p}$ "norm". It can be shown that this definition does not depend on the choice of the function $\varphi$. For $1<p<\infty$, it is well known that $H^{p}\left(\mathbf{R}^{n}\right) \cong L^{p}\left(\mathbf{R}^{n}\right)$, $H^{1}\left(\mathbf{R}^{n}\right) \subset L^{1}\left(\mathbf{R}^{n}\right)$ strictly, and for $0<p<1$ the spaces $H^{p}\left(\mathbf{R}^{n}\right)$ and $L^{p}\left(\mathbf{R}^{n}\right)$ are not comparable. 
The following sentences hold in Hardy spaces $H^{p}\left(\mathbf{R}^{n}\right)$ for $0<p \leq 1$ (see pp. $128-129$ in [4]):

(S1) A bounded compactly supported function $f$ belongs to $H^{p}\left(\mathbf{R}^{n}\right)$ if and only if it satisfies the moment conditions $\int x^{\beta} f(x) d x=0$ for all $|\beta| \leq n\left(p^{-1}-1\right)$.

(S2) If $f \in L^{1}\left(\mathbf{R}^{n}\right) \cap H^{p}\left(\mathbf{R}^{n}\right)$, then $\int x^{\beta} f(x) d x=0$, whenever $|\beta| \leq n\left(p^{-1}-1\right)$ and the function $x^{\beta} f(x)$ is in $L^{1}\left(\mathbf{R}^{n}\right)$.

To obtain our result we will compute explicitly in Section 2 the Fourier transform of the kernel $(|x-s|+|x-t|)^{\alpha-1}$ in the $x$ variable, this allows us to get the following identity

$$
\int_{\mathbf{R}} \mathcal{I}_{\alpha+1}\left(a_{1}, a_{2}\right)(x) d x=\frac{\alpha-1}{\alpha} \iint_{\mathbf{R}^{2}} a_{1}(s) a_{2}(t)|t-s|^{\alpha} d s d t
$$

valid for bounded functions $a_{1}$ and $a_{2}$ having compact support with $\int a_{1}=0$ or $\int a_{2}=0$. Then, from (S2), the counter-example will follow to consider $a_{1} \in H^{1}(\mathbf{R})$ and $a_{2} \in H^{p}(\mathbf{R})$ such that $\iint_{\mathbf{R}^{2}} a_{1}(s) a_{2}(t)|t-s|^{\alpha} d s d t \neq 0$.

Notation. We use the following convention for the Fourier transform in $\mathbf{R}$ $\widehat{f}(\xi)=\int f(x) e^{-i x \xi} d x$. As usual we denote with $\mathcal{S}(\mathbf{R})$ the Schwartz space on $\mathbf{R}$.

\section{Preliminaries}

We start with the following lemma.

Lemma 1. For $0<\alpha<1$ and $s \neq t \in \mathbf{R}$ fixed, let $K_{s, t}^{\alpha}$ be the function defined in $\mathbf{R}$ by

$$
K_{s, t}^{\alpha}(x)=(|x-s|+|x-t|)^{\alpha-1}, \quad x \in \mathbf{R} .
$$

Then

$$
\begin{aligned}
\widehat{K_{s, t}^{\alpha}}(\xi)= & -2^{\alpha} \Gamma(\alpha) \sin \left(\frac{(\alpha-1) \pi}{2}\right) e^{-i \xi\left(\frac{s+t}{2}\right)}|\xi|^{-\alpha}+|t-s|^{\alpha-1} \operatorname{sgn}(t-s) \int_{s}^{t} e^{-i x \xi} d x \\
& -\frac{|t-s|^{\alpha}}{\alpha} e^{-i \frac{(s+t)}{2} \xi} \cos \left(\frac{|t-s| \xi}{2}\right)+\frac{i 2^{\alpha} \xi e^{-i \xi\left(\frac{s+t}{2}\right)}}{\alpha} \int_{0}^{\frac{|t-s|}{2}} x^{\alpha} \sin (x \xi) d x,
\end{aligned}
$$

in the distributional sense.

Proof. First we assume that $s<t$. Then for each $\phi \in \mathcal{S}(\mathbf{R})$ fixed, we have

$$
\begin{aligned}
\left(\widehat{K_{s, t}^{\alpha}}, \phi\right) & =\left(K_{s, t}^{\alpha}, \widehat{\phi}\right)=\int_{\mathbf{R}} K_{s, t}^{\alpha}(x) \widehat{\phi}(x) d x \\
& =\int_{t}^{+\infty} K_{s, t}^{\alpha}(x) \widehat{\phi}(x) d x+\int_{s}^{t} K_{s, t}^{\alpha}(x) \widehat{\phi}(x) d x+\int_{-\infty}^{s} K_{s, t}^{\alpha}(x) \widehat{\phi}(x) d x \\
& =I+I I+I I I .
\end{aligned}
$$

Let us now proceed to compute each one of these integrals,

$$
\begin{aligned}
I & =\int_{\mathbf{R}} \chi_{(t,+\infty)}(x)(2 x-(s+t))^{\alpha-1} \widehat{\phi}(x) d x \\
& =2^{\alpha-1} \int_{\mathbf{R}} x^{\alpha-1} \chi_{\left(\frac{t-s}{2},+\infty\right)}(x)\left(e^{-i(\cdot) \frac{(s+t)}{2}} \phi\right)^{\curlywedge}(x) d x \\
& =2^{\alpha-1} \int_{\mathbf{R}} x_{+}^{\alpha-1}\left(e^{-i(\cdot) \frac{(s+t)}{2}} \phi\right)^{\curlywedge}(x) d x-2^{\alpha-1} \int_{\mathbf{R}} x^{\alpha-1} \chi_{\left(0, \frac{t-s}{2}\right)}(x)\left(e^{-i(\cdot) \frac{(s+t)}{2}} \phi\right)^{\curlywedge}(x) d x \\
& =2^{\alpha-1} \int_{\mathbf{R}} x_{+}^{\alpha-1}\left(e^{-i(\cdot) \frac{(s+t)}{2}} \phi\right)^{\curlywedge}(x) d x
\end{aligned}
$$




$$
-2^{\alpha-1} \int_{\mathbf{R}}\left(\frac{(t-s)^{\alpha} e^{-i \xi \frac{(t-s)}{2}}}{2^{\alpha} \alpha}+\frac{i \xi}{\alpha} \int_{0}^{\frac{t-s}{2}} x^{\alpha} e^{-i x \xi} d x\right) e^{-i \xi \frac{(s+t)}{2}} \phi(\xi) d \xi,
$$

to compute $I I I$ we proceed as in $I$, thus

$$
\begin{aligned}
I I I= & 2^{\alpha-1} \int_{\mathbf{R}} x_{-}^{\alpha-1}\left(e^{-i(\cdot) \frac{(s+t)}{2}} \phi\right) \uparrow(x) d x \\
& -2^{\alpha-1} \int_{\mathbf{R}}\left(\frac{(t-s)^{\alpha}}{2^{\alpha} \alpha} e^{i \xi\left(\frac{t-s}{2}\right)}-\frac{i \xi}{\alpha} \int_{0}^{\frac{t-s}{2}} x^{\alpha} e^{i x \xi} d x\right) e^{-i \xi \frac{(s+t)}{2}} \phi(\xi) d \xi,
\end{aligned}
$$

so

$$
\begin{aligned}
I+I I I= & 2^{\alpha-1} \int_{\mathbf{R}}|x|^{\alpha-1}\left(e^{-i(\cdot) \frac{(s+t)}{2}} \phi\right)^{\wedge}(x) d x \\
& -\int_{\mathbf{R}} \frac{(t-s)^{\alpha}}{\alpha} e^{-i \frac{(s+t)}{2} \xi} \cos \left(\frac{(t-s) \xi}{2}\right) \phi(\xi) d \xi \\
& +\int_{\mathbf{R}}\left(\frac{i 2^{\alpha} \xi e^{-i \xi\left(\frac{s+t}{2}\right)}}{\alpha} \int_{0}^{\frac{(t-s)}{2}} x^{\alpha} \sin (x \xi) d x\right) \phi(\xi) d \xi .
\end{aligned}
$$

Now $I I$ is easy, indeed

$$
I I=\int_{\mathbf{R}} \chi_{(s, t)}(x)(t-s)^{\alpha-1} \widehat{\phi}(x) d x=\int_{\mathbf{R}}\left((t-s)^{\alpha-1} \int_{s}^{t} e^{-i x \xi} d x\right) \phi(\xi) d \xi .
$$

Since

$$
\widehat{|x|^{\alpha-1}}(\xi)=-2 \Gamma(\alpha) \sin \left(\frac{(\alpha-1) \pi}{2}\right)|\xi|^{-\alpha}
$$

(see equation (12), p. 173, in [2]), the lemma follows for the case $s<t$. Finally, exchanging the roles of $s$ and $t$ we obtain the statement of the lemma.

Corollary 2. If $a_{1}$ and $a_{2}$ are two bounded functions on $\mathbf{R}$ with compact support and such that $\int a_{1}=0$ or $\int a_{2}=0$, then

$$
\int_{\mathbf{R}} \mathcal{I}_{\alpha+1}\left(a_{1}, a_{2}\right)(x) d x=\frac{\alpha-1}{\alpha} \iint_{\mathbf{R}^{2}} a_{1}(s) a_{2}(t)|t-s|^{\alpha} d s d t .
$$

Proof. It is easy to check that $\mathcal{I}_{\alpha+1}\left(a_{1}, a_{2}\right)(\cdot) \in L^{1}(\mathbf{R})$. Let $\varphi \in \mathcal{S}(\mathbf{R})$ be an even function such that $\varphi(0)=1$ and for $\epsilon>0$ let $\varphi_{\epsilon}(x)=\varphi(\epsilon x)$. Since

$$
\int_{\mathbf{R}} \mathcal{I}_{\alpha+1}\left(a_{1}, a_{2}\right)(x) d x=\lim _{\epsilon \rightarrow 0^{+}} \int_{\mathbf{R}} \mathcal{I}_{\alpha+1}\left(a_{1}, a_{2}\right)(x) \varphi_{\epsilon}(x) d x,
$$

we will proceed to compute this limit.

$$
\begin{aligned}
\lim _{\epsilon \rightarrow 0^{+}} \int_{\mathbf{R}} \mathcal{I}_{\alpha+1}\left(a_{1}, a_{2}\right)(x) \varphi_{\epsilon}(x) d x & =\lim _{\epsilon \rightarrow 0^{+}} \iint_{\mathbf{R}^{2}} a_{1}(s) a_{2}(t)\left(\int_{\mathbf{R}} K_{s, t}^{\alpha}(x) \varphi_{\epsilon}(x) d x\right) d s d t \\
& =\lim _{\epsilon \rightarrow 0^{+}} \iint_{\mathbf{R}^{2}} a_{1}(s) a_{2}(t)\left(\int_{\mathbf{R}} \widehat{K_{s, t}^{\alpha}}(\xi) \widehat{\varphi} \epsilon(\xi) d \xi\right) d s d t \\
& =\iint_{\mathbf{R}^{2}} a_{1}(s) a_{2}(t) \lim _{\epsilon \rightarrow 0^{+}}\left(\int_{\mathbf{R}} \widehat{K_{s, t}^{\alpha}}(\epsilon \xi) \widehat{\varphi}(\xi) d \xi\right) d s d t \\
& =\frac{\alpha-1}{\alpha} \iint_{\mathbf{R}^{2}} a_{1}(s) a_{2}(t)|t-s|^{\alpha} d s d t
\end{aligned}
$$


where the last equality follows from Lemma 1, the moment condition of $a_{1}$ (or $a_{2}$ ) and that $\varphi(0)=1$.

\section{A counter-example}

We take $a_{1}(s)=\chi_{(-1,0)}(s)-\chi_{(0,1)}(s)$ and $a_{2}(t)=a_{1}(t-2)$. From (S1) it follows that $a_{1} \in H^{1}(\mathbf{R})$ and $a_{2} \in H^{(\alpha+1)^{-1}}(\mathbf{R})$ for each $0<\alpha<1$. A computation gives

$$
\iint a_{1}(s) a_{2}(t)|t-s|^{\alpha} d s d t=\frac{4 \cdot 3^{\alpha+2}-4^{\alpha+2}-6 \cdot 2^{\alpha+2}+4}{(\alpha+1)(\alpha+2)} \neq 0 .
$$

From (S2) and corollary 2 it obtains that $\mathcal{I}_{\alpha+1}\left(a_{1}, a_{2}\right)(\cdot) \notin H^{1}(\mathbf{R})$, for each $0<\alpha<1$. For $0<p<(\alpha+1)^{-1}$ and $\frac{1}{q}=\frac{1}{p}-\alpha$, we take $N$ as any fixed integer with $N>p^{-1}-1$, then the set of all bounded, compactly supported functions for which $\int x^{\beta} f(x) d x=0$, for all $|\beta| \leq N$ is dense in $H^{r}(\mathbf{R})$ for each $p \leq r \leq 1$ (see 5.2 b), pp. 128, in [4]). In particular, there exists $b \in H^{p}(\mathbf{R})$ such that $\left\|a_{1}\right\|_{H^{1}}\left\|a_{2}-b\right\|_{H^{(\alpha+1)^{-1}}}<$ $\left|\int_{\mathbf{R}} \mathcal{I}_{\alpha+1}\left(a_{1}, a_{2}\right)(x) d x\right| / 2 C$. Then

$$
\begin{aligned}
\left|\int_{\mathbf{R}} \mathcal{I}_{\alpha+1}\left(a_{1}, b\right)(x) d x\right| & \geq\left|\int_{\mathbf{R}} \mathcal{I}_{\alpha+1}\left(a_{1}, a_{2}\right)(x) d x\right|-\int_{\mathbf{R}}\left|\mathcal{I}_{\alpha+1}\left(a_{1}, a_{2}-b\right)(x)\right| d x \\
& \geq\left|\int_{\mathbf{R}} \mathcal{I}_{\alpha+1}\left(a_{1}, a_{2}\right)(x) d x\right|-C\left\|a_{1}\right\|_{H^{1}}\left\|a_{2}-b\right\|_{H^{(\alpha+1)}-1} \\
& >\frac{1}{2}\left|\int_{\mathbf{R}} \mathcal{I}_{\alpha+1}\left(a_{1}, a_{2}\right)(x) d x\right|>0,
\end{aligned}
$$

where the second inequality follows from Theorem 1.1 in [1] with $p_{1}=1, p_{2}=$ $(\alpha+1)^{-1}$ and $q=1$. But then the operator $\mathcal{I}_{\alpha+1}$ is not bounded from $H^{1}(\mathbf{R}) \times H^{p}(\mathbf{R})$ into $H^{q}(\mathbf{R})$ for $0<p \leq(\alpha+1)^{-1}$ and $\frac{1}{q}=\frac{1}{p}-\alpha$, since $\int_{\mathbf{R}} \mathcal{I}_{\alpha+1}\left(a_{1}, b\right)(x) d x \neq 0$.

We conclude this note by summarizing our main result in the following theorem.

Theorem 3. For $1<\gamma<2$, let $\mathcal{I}_{\gamma}$ be the multilinear fractional integral operator given by

$$
\mathcal{I}_{\gamma}\left(f_{1}, f_{2}\right)(x)=\iint_{\mathbf{R}^{2}} \frac{f_{1}(s) f_{2}(t)}{(|x-s|+|x-t|)^{2-\gamma}} d s d t, \quad x \in \mathbf{R} .
$$

Then the operator $\mathcal{I}_{\gamma}$ is not bounded from $H^{1}(\mathbf{R}) \times H^{p}(\mathbf{R})$ into $H^{q}(\mathbf{R})$ for $0<p \leq \gamma^{-1}$ and $\frac{1}{q}=1+\frac{1}{p}-\gamma$.

Acknowledgement. I express my thanks to the referee for the useful comments.

\section{References}

[1] Cruz-Uribe, D., K. Moen, and H. V. Nguyen: Multilinear fractional Calderón-Zygmund operators on weighted Hardy spaces. - Preprint: arXiv:1903.01593v1 [math.CA].

[2] Gelfand, I. M., and G. E. Shilov: Generalized functions, properties and operations. Volume 1. - Academic Press Inc., 1964.

[3] Lin, Y., and S. LU: Boundedness of multilinear singular integral operators on Hardy and Herz-type spaces. - Hokkaido Math. J. 36:3, 2007, 585-613.

[4] Stein, E. M.: Harmonic analysis: Real-variable methods, orthogonality, and oscillatory integrals. - Princeton Univ. Press, Princeton, N.J., 1993. 